\title{
Could personalized management of menopause based on genomics become a reality?
}

\author{
"...for the use of genomics to enhance personalized MHT to become a \\ reality, additional resources will be required..."
}

First draft submitted: 21 March 2016; Accepted for publication: 21 March 2016;

Published online: 4 May 2016

Keywords: estrogen $\bullet$ hormone therapy $\bullet$ hormones $\bullet$ menopause

Menopause is an important health issue, ultimately affecting all women. The influence of genetic variation on menopausal physiology is not clearly elucidated, but heritability estimates of the age at menopause range from 31 to $78 \%[1-3]$. In addition, menopause is manifested by a multifactorial phenotype characterized by such diverse symptoms as disturbances of sleep, mood, cognition, temperature regulation (vasomotor symptoms), arthralgias, loss of bone mineral density and vaginal dryness. A single drug, estrogen, is mostly effective in ameliorating these symptoms. However, use of estrogen is not without risks, including increased risk for venous thromboembolism and stroke [4]. However, these risks vary depending upon a woman's age and time since menopause, the presence of other comorbidities, the menopausal hormone therapy (MHT) regimen used (including concomitant use of a progestogen) and the formulation, dose and route of administration $[5,6]$. The questions then become can new tools and techniques to query the human genome be used to optimize selection of a hormone therapy regimen with optimal efficacy and minimal risk, or to identify cases in which nonhormonal treatment approaches are preferred? After all, translating the findings from techniques to query the human genome to clinical practice is the promise of precision medicine.

Pharmacogenomics is one aspect of precision medicine that has the potential to impact all areas of medicine, including
MHT. The goal of pharmacogenomics is to use genetic information to predict how an individual will respond to a drug, with the ultimate objective of aiding clinicians in selecting the right drug, in the right dose, at the right time, for every patient in order to ensure drug efficacy and to avoid adverse drug reactions. Estrogen is the most effective treatment for vasomotor and other symptoms related to menopause, and the current approach to individualizing MHT includes consideration of the severity of the menopausal symptoms, a personalized risk assessment and the patient's personal preferences [5]. Typically, dosing is targeted toward symptom relief, but there is significant variability in the doses required for symptom relief among women. For women experiencing premature ( $<40$ years) or early menopause ( $<45$ years), estrogen therapy is needed not only for symptom management, but also to protect against the potential long-term adverse health consequences of early estrogen deprivation, including increased risk for cardiovascular disease, osteoporosis, dementia, parkinsonism, mood disorders, sexual dysfunction and early death [7]. While the doses or serum levels of estradiol required for bone protection have been determined, and current practice suggests aiming for doses that achieve premenopausal levels, the target dose or perhaps more importantly, the serum or tissue levels needed for brain and heart protection in women with premature or early menopause have yet to be determined [7].

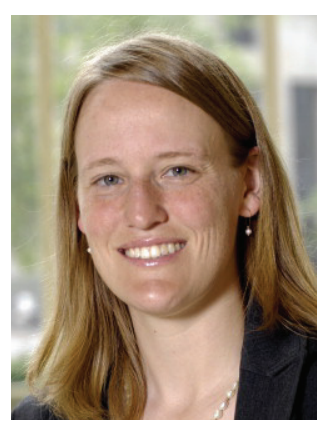

Ann M Moyer

Departments of Pathology \& Laboratory Medicine, Mayo Clinic, 200 First St. SW, Rochester, MN 55905, USA

Virginia M Miller

Author for correspondence: Departments of Surgery \& Physiology \& Biomedical Engineering, Women's Health Research Center, Mayo Clinic, 200 First St. SW, Rochester, MN 55905, USA

Tel.: +1 5072842290

Fax: +1 5072662233

miller.virginia@mayo.edu

Stephanie S Faubion

Departments of General Internal Medicine, Women's Health Clinic, Mayo Clinic, Rochester, MN 55905, USA

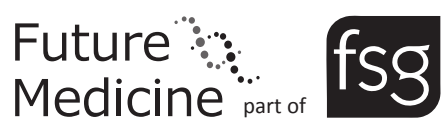


When an exogenous hormone or xenobiotic is administered, genetic variation in both pharmacodynamic and pharmacokinetic pathways may impact the efficacy and toxicity of the drug. On the pharmacodynamics side for estrogens, a major target is the estrogen receptor, which is highly polymorphic and has been the focus of many studies [8]. On the pharmacokinetics side, estrogens can be converted into a storage pool of sulfate-conjugated estrogens by sulfotransferases (SULTs); the process can be reversed by sulfatases. Furthermore, estrogens can be transformed into metabolites by CYP1A1, CYP1A2, CYP1B1 and CYP3A. These metabolites can then further undergo glucuronidation or sulfation, catalyzed by glucoronyltransferases (UGTs) and SULTs, leading to elimination [9]. Many of the enzymes involved in the estrogen metabolism/pharmacokinetic pathway are encoded by genes that are highly polymorphic in human populations.

\section{“...progress has been made in understanding the influence of genetic variation on estrogen efficacy and metabolism..."}

Polymorphisms in genes encoding drug metabolizing enzymes, such as those involved in estrogen metabolism, have been the focus of many pharmacogenomic studies. Based on a woman's genotype, her drug metabolism phenotype for that enzyme can be predicted and may range from poor metabolizer (absent or low enzyme activity) to extensive (average enzyme activity) to ultrarapid (high enzyme activity) (Supplementary Figure 1). Although traditionally pharmacogenomic studies have applied to the administration of exogenous xenobiotics, these same principles can be applied to the metabolism of endogenous and exogenous hormones. While genetics may predict the drug/hormone metabolizer phenotype of a woman, additional variables are also important. Other drugs, foods or chemicals that the individual may be exposed to can also alter the metabolism status by inducing or inhibiting enzymes. Induction can occur either by directly binding and activating an enzyme or by increasing expression of the gene encoding the enzyme, whereas inhibition occurs when a compound blocks or decreases enzyme activity. For example, CYP3A4 can be either induced or inhibited. Phenobarbital is known to induce CYP3A4, while grapefruit can inhibit the enzyme [10]. Furthermore, CYP1A1 and CYP1A2 can be induced by aryl hydrocarbons, such as those found in cigarette smoke [11]. Although the impact of variation in each individual enzyme on estrogen metabolism can be studied in model systems, due to the number of polymorphic enzymes involved and the additional variation that may be introduced by other drugs/environmental factors, it becomes quite complex to study the influence of genetic variation on estrogen metabolism in vivo.

The heterogeneity in the experience of menopause involves several aspects, including the type and timing of menopause (e.g., premature, early, natural at the average age or surgical), the type of symptoms experienced (e.g., vasomotor, cognitive, sleep, mood, joint, sexual) as well as symptom severity and duration. There is also significant variability in the duration of vasomotor symptoms experienced by women. For example, the mean duration of symptoms is approximately 7 years, but over $30 \%$ of women will experience moderately severe symptoms for 10 years or more [12,13]. Additionally, how women perceive and experience their symptoms is known to vary depending on race/ethnicity and culture [14].

While genetic alterations may account for some of the variation in menopausal timing and symptoms, this area has not yet undergone thorough study. In a candidate gene study, variation in SULT1A1, which is a polymorphic gene including both single nucleotide polymorphisms and copy number variation, was evaluated for association with menopausal timing and symptoms, as well as with estrogen levels. Having a higher activity genotype (gene duplication of the higher activity ' $G$ ' allele at rs9282861) was associated with earlier age at menopause, decreased frequency of night sweats and less severe insomnia [15]. Furthermore, relationships between genotypes and estrogen levels were identified within the group of women assigned to oral estrogens, but not other formulations [15]. These data suggest that the influence of genetic variation differs by treatment modality, but due to the small size of this population, will require follow-up in larger cohorts.

Environmental and biological factors may also impact menopausal symptoms, including body mass index, tobacco, alcohol or caffeine use [16], stress, anxiety, a history of recent abuse [17] or adverse childhood experiences [18]. Further work is required to understand the mechanisms by which these environmental and biologic factors affect menopausal symptoms. They may be independent variables or may be intertwined with genetic variation in gene-environment interactions.

Although observational studies suggested that MHT would be cardioprotective, randomized prospective studies are adding clarity and helping to define the subgroups of women who might obtain cardiovascular benefit as well as providing insight into the formulations and routes of administration of MHT that might provide the maximal benefit while reducing the risks [19-23]. A number of studies have approached this problem by analysis of genetic fac- 
tors, but until recently, the assessment of cardiovascular disease and thrombotic risk together as a complex genetic trait had not been studied. Although this type of analysis requires substantially more participants and is more complex, it recapitulates the biology in an individual - with many genes interacting - much better than the study of individual genes and genetic traits. In a recent study, genetic variants in 764 genes involved in thrombosis were evaluated for association with subclinical cardiovascular disease prior to MHT, as defined by carotid artery intima-media thickness and coronary artery calcification. A number of genes of interest were identified, including MAP4K4, IL5, CCL5, SERPINA1 and ABO [24]. A similar study utilizing the same cohort was performed after 4 years of randomization to oral conjugated equine estrogens, transdermal $17 \beta$-estradiol or placebo. In the followup study, several SNPs within the innate immunity pathway were found to alter the treatment effect on 4-year change in carotid intima-medial thickness, and the degree of alteration differed by treatment regimen (i.e., oral conjugated equine estrogen or transdermal $17 \beta$-estradiol) [25]. While genetic variants in these genes of the innate immunity pathway may be promising as biomarkers for risk of developing accelerated cardiovascular disease, this study emphasizes the need to account for hormonal status in other larger cohorts investigating similar genetic associations with complex disease processes. In addition, other mechanistic studies will be required to understand these interactions within complex phenotypes in order to begin to build them into clinical algorithms for use in optimizing MHT.

Pharmacogenomic approaches may help identify women with different estrogen dose requirements based on identification of genetic variants in enzymes involved in hormone/drug metabolism and impact-

\section{References}

1 Morris DH, Jones ME, Schoemaker MJ, Ashworth A, Swerdlow AJ. Familial concordance for age at natural menopause: results from the Breakthrough Generations Study. Menopause 18(9), 956-961 (2011).

2 Murabito JM, Yang Q, Fox C, Wilson PW, Cupples LA. Heritability of age at natural menopause in the Framingham Heart Study. J. Clin. Endocrinol. Metab. 90 (6), 3427-3430 (2005).

3 Van Asselt KM, Kok HS, Pearson PL et al. Heritability of menopausal age in mothers and daughters. Fertil. Steril. 82(5), 1348-1351 (2004).

4 Rossouw JE, Anderson GL, Prentice RL et al. Risks and benefits of estrogen plus progestin in healthy postmenopausal women: principal results from the Women's Health Initiative randomized controlled trial. JAMA 288(3), 321-333 (2002). ing hormone/drug targets. Although progress has been made in understanding the influence of genetic variation on estrogen efficacy and metabolism through candidate gene studies, due to the complexity of both the estrogen pharmacodynamic and pharmacokinetic pathways, and the many additional variables reviewed here that may be of importance, large studies will be required to develop genetically based algorithms for estrogen administration/dosing. Furthermore, it will likely be important to consider other medications and environmental factors if such algorithms are to be developed in the future. Advances in research regarding the pharmacogenetics and pharmacogenomics of estrogen metabolism may allow for more personalized use of MHT for management of menopausal symptoms. However, for the vision for the use of genomics to enhance personalized MHT to become a reality, additional resources will be required to perform the necessary research to further develop these promising research findings into information that is clinically actionable.

\section{Supplementary data}

To view the supplementary data that accompany this paper, please visit the journal website at: www.futuremedicine.com/ doi/full/10.2217/pgs.16.17

\section{Financial \& competing interests disclosure}

The authors have no relevant affiliations or financial involvement with any organization or entity with a financial interest in or financial conflict with the subject matter or materials discussed in the manuscript. This includes employment, consultancies, honoraria, stock ownership or options, expert testimony, grants or patents received or pending, or royalties.

No writing assistance was utilized in the production of this manuscript

5 Kaunitz AM, Manson JE. Management of menopausal symptoms. Obstet. Gynecol. 126(4), 859-876 (2015).

6 Sood R, Faubion SS, Kuhle CL, Thielen JM, Shuster LT. Prescribing menopausal hormone therapy: an evidence-based approach. Int. J. Womens Health 6, 47-57 (2014).

7 Faubion SS, Kuhle CL, Shuster LT, Rocca WA. Long-term health consequences of premature or early menopause and considerations for management. Climacteric 18(4), 483-491 (2015).

8 Kallel I, Rebai M, Rebai A. Mutations and polymorphisms of estrogens receptors genes and diseases susceptibility. J. Recept. Signal. Transduct. Res. 32(6), 304-313 (2012).

9 Zeruesenay D, Nguyen A, Flockhart D et al. Estrogen metabolism pathway (6 March 2016). www.pharmgkb.org

10 He K, Iyer KR, Hayes RN, Sinz MW, Woolf TF, Hollenberg $\mathrm{PF}$. Inactivation of cytochrome P450 3A4 by bergamottin, 
a component of grapefruit juice. Chem. Res. Toxicol. 11(4), 252-259 (1998).

11 Ma Q, Lu AY. CYP1A induction and human risk assessment: an evolving tale of in vitro and in vivo studies. Drug Metab. Dispos. 35(7), 1009-1016 (2007).

12 Avis NE, Crawford SL, Greendale G et al. Duration of menopausal vasomotor symptoms over the menopause transition. JAMA Intern. Med. 175(4), 531-539 (2015).

13 Freeman EW, Sammel MD, Sanders RJ. Risk of long-term hot flashes after natural menopause: evidence from the Penn Ovarian Aging Study cohort. Menopause 21(9), 924-932 (2014).

14 Gold EB, Colvin A, Avis N et al. Longitudinal analysis of the association between vasomotor symptoms and race/ethnicity across the menopausal transition: study of women's health across the nation. Am. J. Public Health 96(7), 1226-1235 (2006).

15 Moyer AMDAM, Weinshilboum RM, Miller VM. Influence of SULT1A1 genetic variation on age at menopause, estrogen levels, and response to hormone therapy in recently menopausal white women. Menopause (2016) (In Press).

16 Faubion SS, Sood R, Thielen JM, Shuster LT. Caffeine and menopausal symptoms: what is the association? Menopause 22(2), 155-158 (2015).

17 Vegunta S, Kuhle C, Kling JM et al. The association between recent abuse and menopausal symptom bother: results from the Data Registry on Experiences of Aging, Menopause, and Sexuality (DREAMS). Menopause PMID: 26783985 (2016) (Epub ahead on print).

18 Thurston RC, Bromberger J, Chang Y et al. Childhood abuse or neglect is associated with increased vasomotor symptom reporting among midlife women. Menopause 15(1), 16-22 (2008).

19 Bush TL. Evidence for primary and secondary prevention of coronary artery disease in women taking oestrogen replacement therapy. Eur. Heart. J. 17(Suppl. D), 9-14 (1996).

20 Canonico M, Oger E, Plu-Bureau G et al. Hormone therapy and venous thromboembolism among postmenopausal women: impact of the route of estrogen administration and progestogens: the ESTHER study. Circulation 115(7), 840-845 (2007).

21 Miller VM. Estrogen metabolomics: a physiologist's perspective. Hypertension 56(5), 816-818 (2010).

22 Miller VM, Manson JE. Women's Health Initiative Hormone Therapy Trials: new insights on cardiovascular disease from additional years of follow up. Curr. Cardiovasc. Risk Rep. 7(3), 196-202 (2013).

23 Winham SJ, De Andrade M, Miller VM. Genetics of cardiovascular disease: importance of sex and ethnicity. Atherosclerosis 241(1), 219-228 (2015).

24 Miller VM, Petterson TM, Jeavons EN et al. Genetic polymorphisms associated with carotid artery intima-media thickness and coronary artery calcification in women of the Kronos Early Estrogen Prevention Study. Physiol. Genomics 45(2), 79-88 (2013).

25 Miller VM, Jenkins GD, Biernacka JM et al. Pharmacogenomics of estrogens on changes in carotid artery intima-medial thickness and coronary arterial calcification: Kronos Early Estrogen Prevention Study. Physiol. Genomics 48(1), 33-41 (2016). 